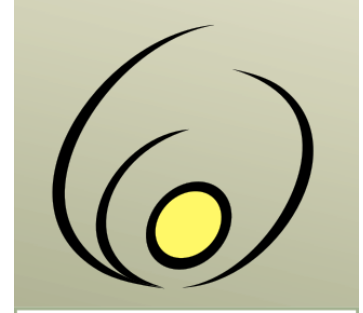

Fórum de Pró-Reitores de Extensão das Instituições Públicas de Educação Superior Brasileiras

\section{Revista Brasileira de Extensão Universitária}

v. 11 , n. 1, p. 61-71, jan.-abr. 2020

e-ISSN 2358-0399

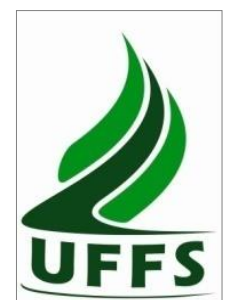

Content shared under Creative Commons Attribution 4.0 Licence CC-BY

\title{
A microbiologia e a extensão universitária
}

\author{
Belize Rodrigues Leite $^{1}$, Patricia Valente ${ }^{2}$
}

\begin{abstract}
Resumo: Conhecimentos sobre Microbiologia promovem o cuidado pessoal, para com a saúde e o meio ambiente. Isso contribui, em uma perspectiva mais ampla, para a formação de seres mais conscientes de sua realidade. A extensão configura-se como uma das finalidades da Universidade, por meio da qual institui práticas cidadãs de pesquisa e ensino direcionadas à comunidade em geral. O presente estudo teve como objetivo analisar o perfil das ações de extensão universitária que abordam temas da Microbiologia, baseando-se na produção científica nacional das últimas duas décadas. Tais ações de extensão foram encontradas em 109 publicações deste período. Predominaram ações na área da Microbiologia Clínica (40/109), destacando-se temas como Educação e Promoção da saúde (32/109 e 22/109, respectivamente). Entre as doenças abordadas nestas ações, as Viroses (13/109) foram preponderantes frente às Parasitoses (3/109) e as Micoses (2/109). O ensino também tem sido o foco das ações de extensão em Microbiologia, uma vez que a maior parte delas desenvolveu ou aprimorou Práticas educativas (29/109), atendendo majoritariamente ao público escolar (42\%). As universidades públicas participam massivamente destas ações, representando $91 \%$ das iniciativas. As informações geradas retornaram aos participantes em $74 \%$ das ações analisadas, demonstrando a importância da extensão para veicular conhecimentos científicos em diferentes públicos. Este estudo pode contribuir para que as ações voltadas à Microbiologia cresçam, se fortaleçam e aumentem ainda mais a sua significância social.
\end{abstract}

Palavras-chave: Ensino de microbiologia; Educação; Saúde; Ambiente

\section{The microbiology and the university extension}

Abstract: Knowledge about Microbiology promotes personal and health care, in addition to the care for the environment. This knowledge contributes, in a broader perspective, to the formation of beings more aware of their reality. One of the purposes of the University, the extension is the set of research and teaching practices directed to the community in general. This study aimed to analyze the profile of university extension actions involving Microbiology themes, based on the national scientific production of the last two decades. These extension actions were found in 109 publications of this period. Actions in the Clinical Microbiology area predominated (40/109), highlighting topics such as Education and Health Promotion (32/109 and 22/109, respectively). Among the diseases addressed in these actions, Viruses (13/109) were preponderant against Parasitic infections (3/109) and Mycoses (2/109). Teaching has also been the focus of extension actions in Microbiology, since most of them have developed or improved educational practices (29/109), supporting mainly the students (42\%). Public universities participate massively in these actions, representing $91 \%$ of initiatives. The information generated returned to the participants in $74 \%$ of the actions analyzed, demonstrating the importance of extension to convey scientific knowledge in different audiences. This study may contribute to the actions aimed at Microbiology to grow, strengthen, and further increase their social significance.

Keywords: Microbiology teaching; Education; Health; Environment
Originais recebidos em

11 de outubro de 2019

Aceito para publicação em

03 de março de 2020

1

Universidade Federal do Rio Grande do Sul, Porto Alegre/RS.

belize.leite@gmail.com

(autora para correspondência)

2 Universidade Federal do Rio Grande do Sul, Porto Alegre/RS. patricia.valente@ufrgs.br 


\section{Introdução}

A UNESCO (Organização das Nações Unidas para a Educação, a Ciência e a Cultura) reconhece que as desigualdades entre pobres e ricos não está relacionada somente a bens materiais, mas também ao acesso ao conhecimento científico (Carvalho, 2005). Um relatório divulgado pelo Programa das Nações Unidas para o Desenvolvimento apontou que o Brasil conseguiu reduzir radicalmente seus índices de pobreza entre os anos 2000 e 2014 (Programa das Nações Unidas para o Desenvolvimento, 2014). Neste mesmo período, no Brasil, a proporção de pessoas com idade entre 25 e 34 anos que possuíam ensino superior passou de $8,1 \%$ para 15,2\% (Ferreira, 2016). O crescimento concomitante de ambos os índices, de economia e de ensino, não ocorreu ao acaso. A Universidade é uma instituição que reproduz o modelo de sociedade no qual está inserida, e apresenta grande potencial para conservar ou mesmo transformar este modelo (Chauí, 2001; Fagundes, 1986).

Uma vez que o mundo globalizado é regido por rápidas transformações, principalmente no que diz respeito à tomada de conhecimentos e ao desenvolvimento tecnológico, à Universidade cabe o papel de formar lideranças comprometidas com as necessidades da sociedade, bem como com o seu progresso (Ferreira, 2016). A Extensão está prevista na Constituição brasileira como uma das finalidades da Universidade, objetivando instituir práticas cidadãs de pesquisa e ensino. Corresponde ao conjunto de atividades acadêmicas (educativas, científicas e culturais) voltadas à comunidade em geral, e é responsável por ampliar as relações entre a Universidade e a coletividade. Esta interação possibilita a construção de novos saberes científicos à medida que docentes, discentes e técnicos administrativos trazem um aprendizado construído fora da Universidade que é submetido à reflexão teórica e retorna à sociedade por intermédio da Extensão (Teixeira \& De Araújo, 2018).

A Microbiologia é a área da Biologia que se dedica ao estudo dos microrganismos, seres microscópicos tais como bactérias, fungos, protozoários e vírus. Pesquisa a forma, a estrutura, a reprodução, a fisiologia, o metabolismo e a identificação destes seres. Também investiga sua distribuição natural, as relações ecológicas com os humanos e outros seres vivos, e as alterações físicas e químicas que provocam no meio ambiente (De Carvalho, 2010). Deste modo, a Microbiologia agrega conhecimentos relacionados à importância do cuidado pessoal e para com a saúde e a natureza; contribuindo para a formação de seres mais conscientes de sua realidade e críticos diante da tomada de decisões nestas áreas. Há muito tempo, a Microbiologia deixou de ser tema restrito às salas de aula do ensino superior, ou a laboratórios de pesquisa, para ser tema relacionado às questões básicas de cidadania, envolvendo hábitos do cotidiano, a higiene, o ambiente entre outros (Prado et al., 2004). No entanto, de um modo geral, não é evidente a forma como a Microbiologia vem se inserindo no contexto brasileiro da Extensão Universitária. O objetivo do presente estudo foi analisar o perfil das ações de extensão universitária que abordam temas da Microbiologia, baseando-se na produção científica nacional das últimas duas décadas.

\section{Procedimentos Metodológicos}

Realizou-se ampla pesquisa bibliográfica sobre o tema proposto. Inicialmente, utilizou-se o buscador Google Scholar para explorar o tema de modo mais genérico. Utilizaram-se palavras-chave tais como 'extensão universitária', 'microbiologia', 'bactéria', 'fungo', 'vírus', 'microscópio', 'vacina' e 'doença'. Adicionalmente, de modo mais refinado, estas expressões também foram pesquisadas em 50 revistas científicas apreciadas pela Rede Nacional de Extensão (2017). Uma vez que este estudo objetivou avaliar ações de extensão que transcendam o âmbito da Universidade, foram excluídas da análise pesquisas direcionadas ao público universitário (alunos e professores), como, por exemplo, o desenvolvimento de sítios da internet, cartilhas ou recursos didáticos que visem à melhora do desempenho em uma área ou disciplina específica. 
Visto o caráter multidisciplinar da Microbiologia, houve a necessidade de agrupar os estudos consultados em algumas categorias. Os estudos foram classificados conforme as áreas, os temas e as especificidades abordadas, dentro do contexto da Microbiologia. Os estudos foram subdivididos nas áreas: Microbiologia Clínica (humana), Microbiologia Clínica (veterinária), Microbiologia Ambiental, Microbiologia Agrícola, Microbiologia de Solos, Microbiologia de Alimentos, Microbiologia Aquática, Microbiologia Geral, e Biossegurança - campo multidisciplinar e transversal do conhecimento (Teixeira \& Valle, 2010) que, neste estudo, foi considerado como uma área à parte.

O conceito de 'Promoção da saúde' refere-se a ações de enfrentamento e resolução de questões sanitárias (Buss, 2003), enquanto o de 'Educação em Saúde' relaciona-se a experiências sistemáticas de aprendizagem que facilitem tais ações em saúde (Candeias, 1997). Depreende-se, portanto, que a 'Educação em Saúde' é um pilar essencial da 'Promoção da saúde'. Na presente revisão o enquadramento dos resultados deu-se do seguinte modo: as ações com foco na prevenção de doenças, as quais ocorreram majoritariamente no contexto escolar, foram categorizadas no tema 'Educação em saúde'; enquanto as ações que envolvem uma situaçãoproblema ou portadores de alguma doença e atendem a públicos diferentes do escolar (comunidade, pacientes e usuários de algum serviço ou programa de saúde, por exemplo) foram agrupadas no tema 'Promoção da saúde'.

O tema 'Ensino-aprendizagem' agrupa estudos dedicados ao ensino-aprendizagem de diversas áreas da Microbiologia, e ao desenvolvimento de práticas e recursos didáticos, também realizados em contexto escolar. Estudos com a temática 'Segurança do alimento' são direcionados à avaliação da qualidade de alimentos ou à capacitação de profissionais da área. A temática 'Agropecuária' é atribuída a estudos sobre doenças em animais de corte, enquanto 'Fitossanidade' é o tema envolvendo estudos sobre doenças de plantas ou manejos sustentáveis, como é o caso da agroecologia (Farias et al., 2017; Júnior et al., 2018).

As especificidades dos trabalhos foram divididas em: práticas educativas; recursos didáticos; memorial; infecções sexualmente transmissíveis (IST); viroses; micoses; parasitoses; saúde bucal; doenças negligenciadas; abordagem assistencial; boas práticas; doenças transmitidas por alimentos (DTAs); zoonoses; agroecologia; e tecnologia. O tipo 'Memorial' reuniu estudos que descreviam ações de extensão ao longo da história de um instituto ou núcleo de pesquisas. Estudos sobre doenças virais foram segregados em dois grupos: um específico sobre IST e outro sobre as demais viroses abordadas (Febre amarela, Dengue e Chikungunya). Quanto às 'doenças negligenciadas', incluem-se Doença de Chagas, Leishmaniose e Hanseníase. O caráter 'assistencial' foi atribuído a estudos que prestavam algum tipo de serviço que não se encaixava nas demais especificidades pré-definidas.

A tipificação dos estudos, sob o ponto de vista da extensão universitária, foi adaptada de Fonseca e Lorenzo (2004). Os autores classificam as ações em extensão universitária em cinco tipos genéricos: atividades assistenciais; eventos artísticos, culturais e esportivos; atividades de disseminação do conhecimento; prestação de serviços; e transferência de tecnologia. Observou-se que o formato 'prestação de serviços' nem sempre é evidente na literatura. Além disso, a maior parte dos serviços prestados demanda atendimentos ou orientações, pressupostos básicos da categoria 'assistencial', de acordo com os autores. Deste modo, o presente estudo incluiu a 'prestação de serviços' dentro da categoria 'assistencial'.

\section{Resultados}

Foram encontrados 109 estudos que se configuram em ações de extensão universitária que abordam temas da Microbiologia. Os estudos foram publicados entre 2002 e maio de 2019. Estes dados estão listados na íntegra no material suplementar (Tabela S1). 
As áreas da Microbiologia mais bem representadas foram a Microbiologia Clínica (humana e animal) e a Microbiologia Geral (Figura 1), correspondendo a aproximadamente $70 \%$ do total dos estudos avaliados. Os temas Educação em saúde, Ensino-aprendizagem e Promoção da saúde foram abordados pela maior parte destes estudos (Figura 2). Entre os assuntos mais específicos (Figura 3), ações em extensão universitária com foco em práticas educativas foram majoritárias, categoria que foi representada por $26,6 \%$ destes estudos.

Quase metade dos estudos avaliados (45,87\%) foi realizada na região Sudeste do Brasil (Figura 4A), 91\% destes por iniciativa de Universidades públicas (Figura 4B). Prevalecem as ações de extensão de cunho informativo ('disseminação do conhecimento') (Figura 4C), sendo que 56\% destas ações não utilizaram a estrutura da Universidade para cumprir seus objetivos (Figura 4D). O público preponderante é o dos estudantes, alvo de $42 \%$ destes estudos (Figura 4E). Embora $58 \%$ das ações de extensão analisadas venham atendendo a demandas de áreas urbanas, é perceptível que áreas rurais (28\%) ou de difícil acesso (10\%) também venham sendo contempladas (Figura 4F). Predominam os estudos que utilizam métodos diferentes de entrevistas ou questionários: $68 \%$ em detrimento dos 32\% que utilizam (Figura $4 \mathrm{G}$ ). Verificou-se também que $74 \%$ destes estudos contribuíram com um retorno informativo aos participantes das pesquisas (Figura $4 \mathrm{H}$ ).

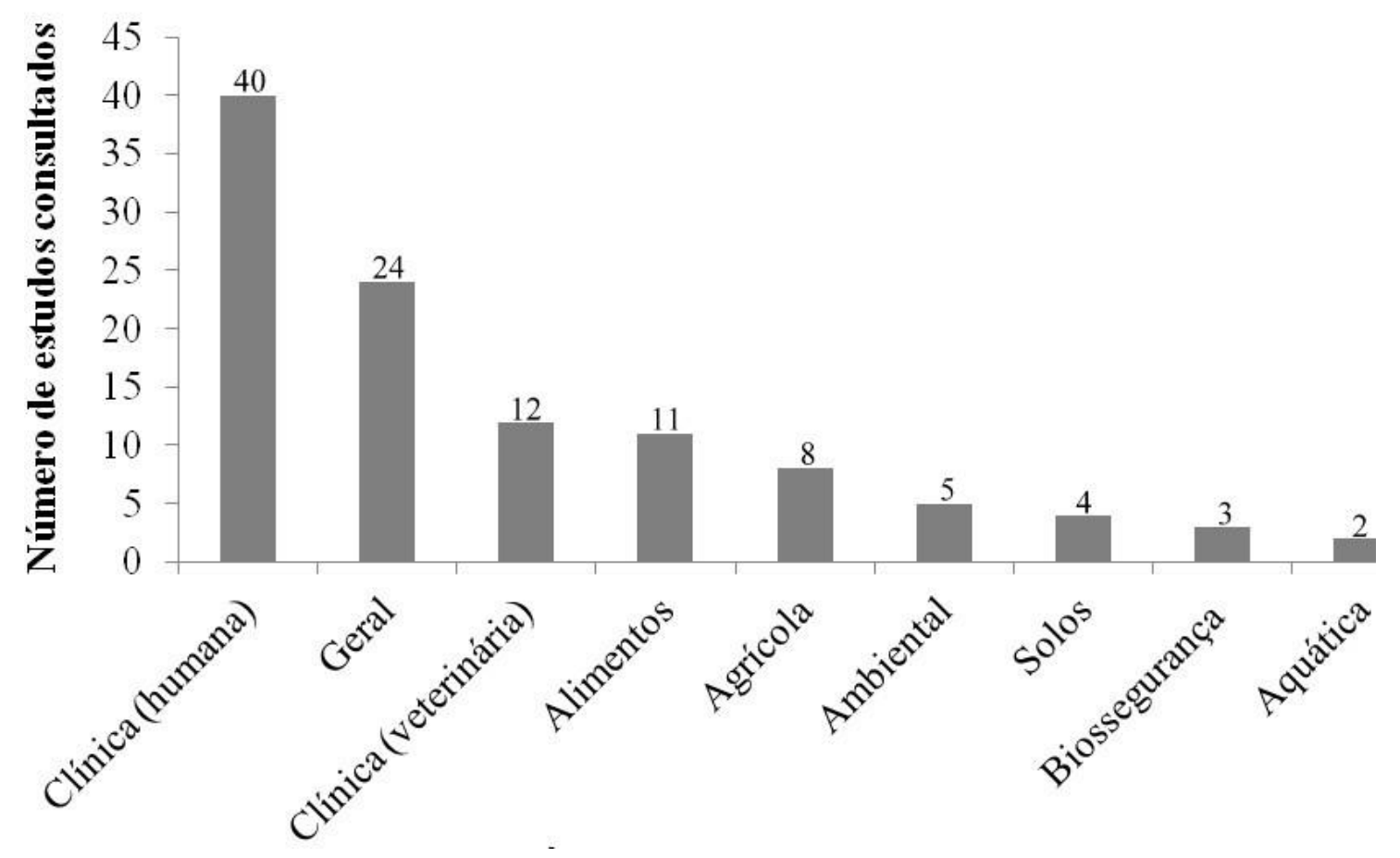

Figura 1. Áreas da Microbiologia representadas em ações de extensão universitária, no período de 2002 a maio de 2019. Os números acima das barras referem-se à quantidade de ações correspondente a cada área. 


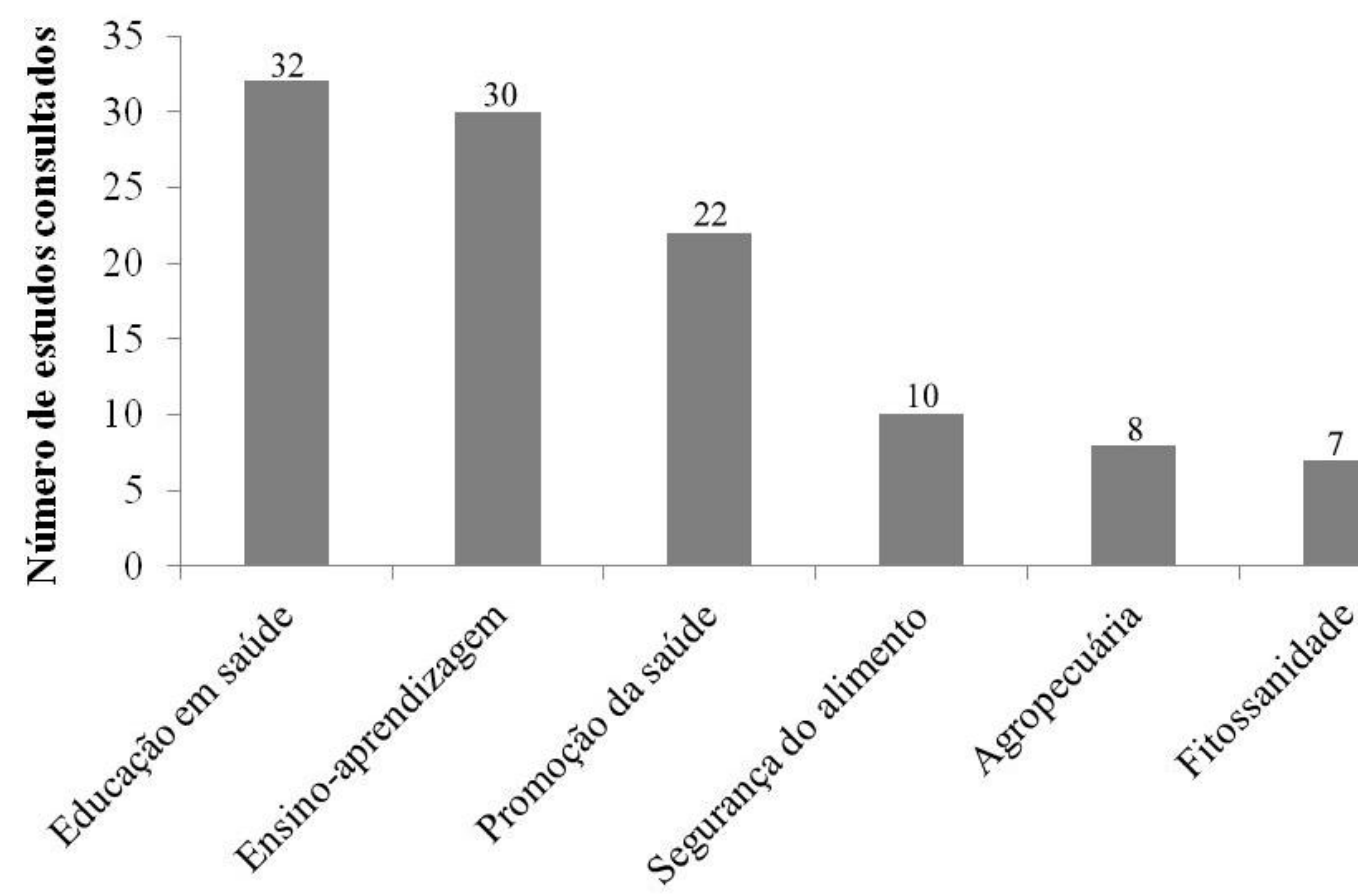

Figura 2. Temática das ações de extensão universitária que abordam a Microbiologia, no período de 2002 a maio de 2019. Os números acima das barras referem-se à quantidade de ações correspondente a cada tema.

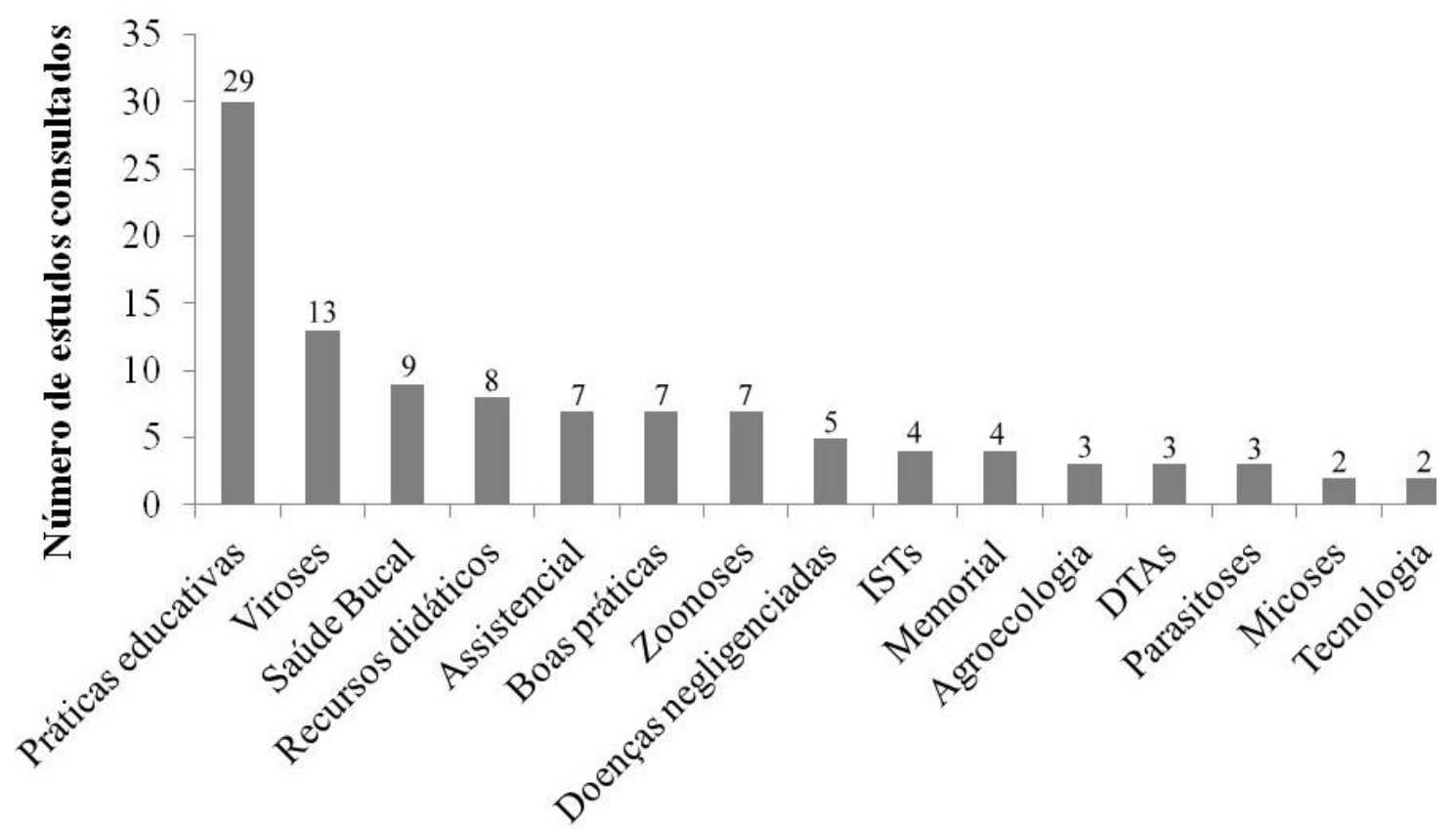

Figura 3. Especificidades das ações de extensão universitária que abordam a Microbiologia, no período de 2002 a maio de 2019. Os números acima das barras referem-se à quantidade de ações correspondente a cada especificidade. 


\section{Regiões contempladas}

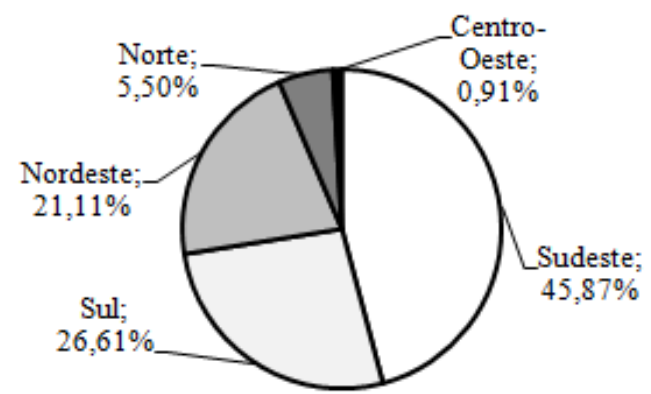

A)

Tipo de estudo

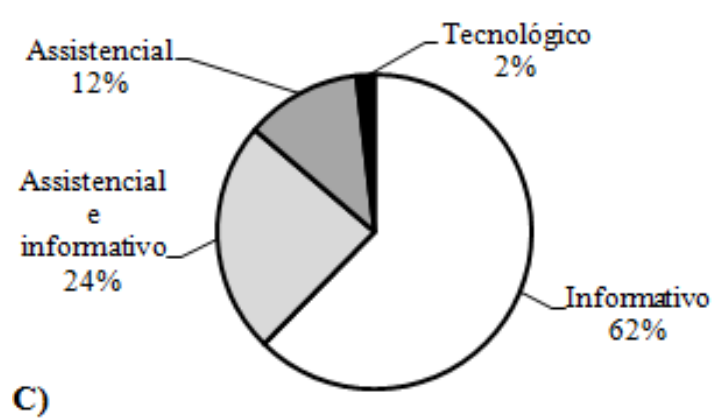

Público alvo

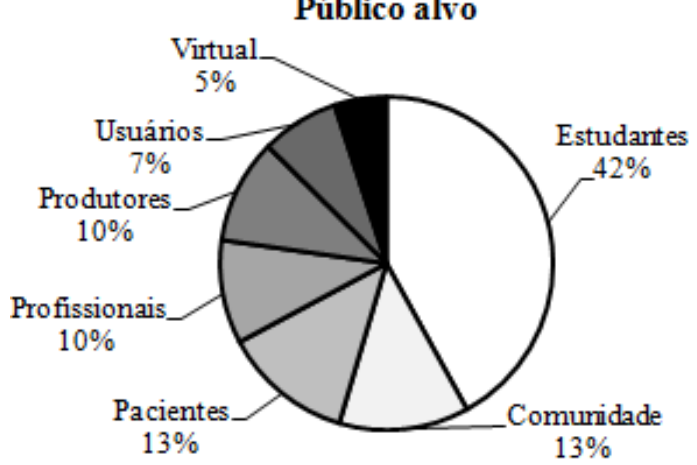

E)

\section{Uso de questionário ou entrevista}

G)

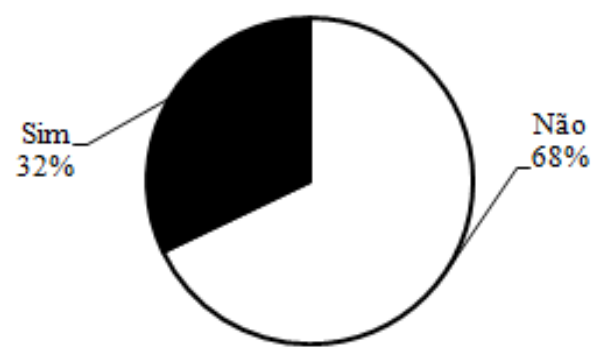

Natureza das instituições executoras

B)

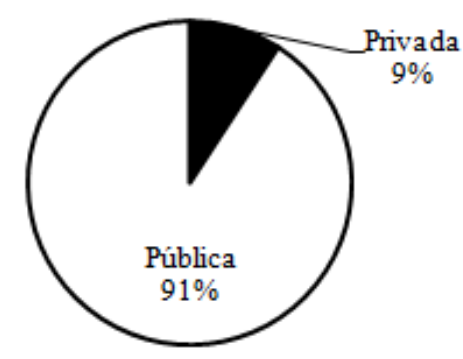

Uso da estrutura da Universidade

D)

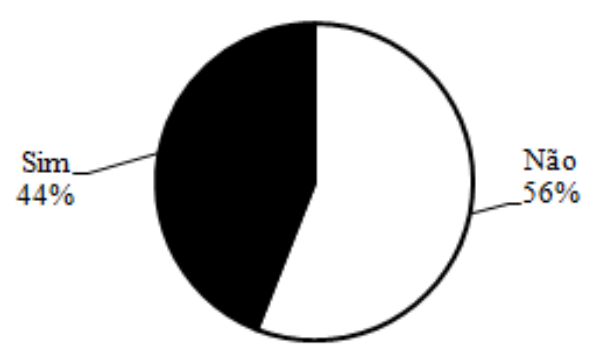

Alcance dos estudos consultados

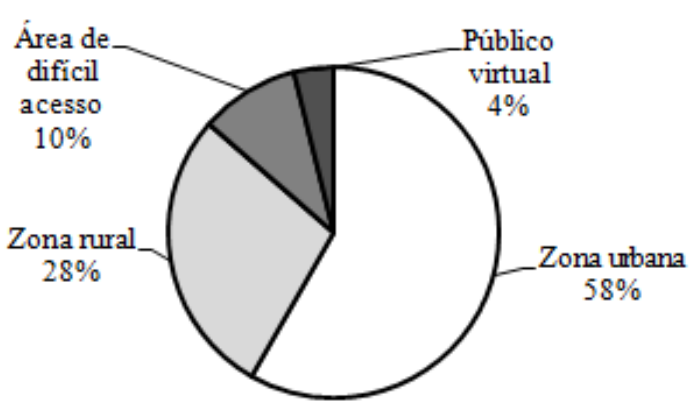

F)

\section{Retorno informativo à comunidade}

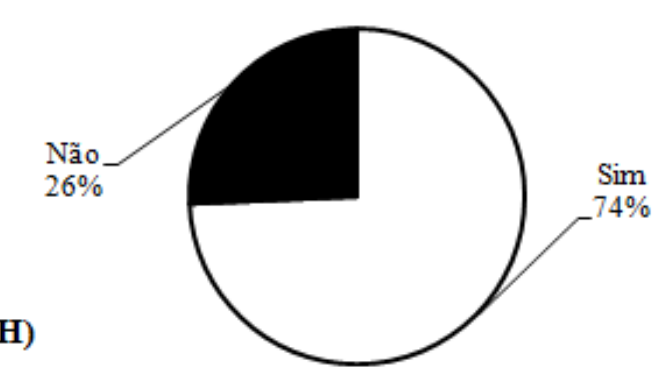

Figura 4. Caracterização das ações em extensão universitária que abordam a Microbiologia quanto à região onde ocorreu o estudo $(\mathbf{A})$, à natureza das instituições executoras $(\mathbf{B})$, ao tipo de estudo $(\mathbf{C})$, ao uso da estrutura da Universidade (D), ao público alvo (E), ao alcance dos estudos consultados (F), ao uso de questionários (G) e ao retorno informativo à comunidade envolvida com o estudo $(\mathbf{H})$. 


\section{Discussão}

Visto o caráter multidisciplinar da Microbiologia, pesquisar e organizar as categorias dos estudos acessados demandou uma análise criteriosa do conteúdo de cada um deles. Observou-se que a Microbiologia é uma área capaz de dialogar com ações de extensão universitária dos mais variados tipos. Contudo, estudos voltados à Microbiologia Clínica foram prevalentes (40/109) (Figura 1). A área clínica destaca-se na produção científica brasileira desde seus primórdios, pois, no país, a pesquisa em saúde baseia-se no modelo biológico, o qual valoriza o enfoque epidemiológico, a ecologia de vetores e a avaliação de risco no que se refere à poluição ambiental (Vieira\& Oliveira, 2011). Consonante a isso, os temas 'Educação em Saúde' e 'Promoção da Saúde' (Figura 2) também foram mais frequentes entre os estudos avaliados (32/109 e 22/109, respectivamente). A maior ocorrência de estudos voltados à 'Educação em Saúde' indica uma maior preocupação em prevenir doenças ao invés de remediá-las (já que os casos que tratam de uma situação-problema foram agrupados no tema 'Promoção da Saúde').

Entre as doenças abordadas nestas ações, as Viroses (13/109) foram preponderantes frente às Parasitoses (3/109) e as Micoses (2/109). Além das Viroses serem o quadro clínico mais comum em pronto atendimentos, o grupo dos Vírus apresenta enorme variabilidade genética: reportou-se que mamíferos podem veicular mais de 320.000 tipos diferentes de vírus (Anthony et al., 2013). A frequência do registro de Viroses bem como a quantidade de tipos de Vírus pode estar relacionada com o protagonismo deste grupo nas ações de extensão. Parasitoses e Micoses estão entre as doenças reconhecidas como negligenciadas pela Organização mundial da Saúde (OMS), percebendo-se um esforço a nível mundial para que estas enfermidades (e também seus agentes) sejam melhor estudadas (Organização Mundial das Nações Unidas, 2015).

Fonseca e Lorenzo (2004) constataram que diferentes universidades apresentam uma grande variedade de critérios de enquadramento do que sejam as atividades de extensão. Oda (2012) verificou que a maior parte dos professores universitários assume realizar atividades de extensão, mas estes demonstram distintas compreensões quando questionados sobre a natureza destas ações, as quais assumem um caráter demasiadamente genérico. Sendo assim, além dos temas, consideramos a necessidade de apreciar as especificidades das ações, categorias ilustradas na Figura 3. Mesmo a saúde sendo uma área preponderante sobre as demais (Figuras 1 e 2), as 'Práticas Educativas' destacaram-se entre as especificidades das ações de extensão (29/109) (Figura 3). Ocorre é que muitos destes estudos abordam a temática saúde, como é o caso, por exemplo, de Korb e colaboradores (2011), que avaliaram os conhecimentos em biologia na educação em saúde; Da Fonseca e colaboradores (2011), que mediaram ações educativas para profissionais de um laboratório de análises clínicas; e de estudos como os de Moresco e colaboradores (2015) e Oliveira e colaboradores (2016), que utilizaram a higienização das mãos como tema gerador de discussões em Microbiologia, dentre outros.

Os resultados demonstraram que as regiões Centro-Oeste e Norte carecem de iniciativas de extensão em Microbiologia (Figura 4A). Nestas regiões é comum a realização de atividades de extensão que são idealizadas por universidades de outras localidades, como é o caso da pesquisa de De Magalhães Bastos e colaboradores (2010), a qual ocorreu por intermédio de um núcleo de Odontologia da Universidade de São Paulo radicado em Roraima. Isso indica que as Universidades não se limitam somente a suprir demandas específicas de suas regiões. Também foram encontradas ações conjuntas de diferentes Universidades ou instituições, tais como a do estudo de Dos Santos e colaboradores (2017), que reuniu esforços da Universidade e do Instituto Federais do Rio Grande do Norte (RN) e da Universidade Federal Rural do Semiárido para disseminar jogos didáticos, com o objetivo de prevenir o vetor da dengue.

A Figura 4B mostra que as Universidades públicas assumem o protagonismo no que diz respeito às ações em extensão universitária que versam sobre temas da Microbiologia (91\%). Estas ações têm atendido inclusive a 
demandas de comunidades de áreas rurais (28\%) (Granato et al., 2015; Da Silva et al., 2017) ou de difícil acesso (10\%) (Mesquita et al., 2013; Sachet \& Arruda, 2018), público geralmente carente de outros tipos de serviços assistenciais (Figura 4F). Atenta-se que, de um modo geral, a continuidade das ações de extensão no Brasil depende diretamente da garantia de investimentos no ensino superior público. Ou seja, reduzir a receita das Universidades públicas implica em distanciá-las ainda mais da comunidade externa.

Apesar da maior parte dos estudos avaliados (62\%) serem de cunho educacional (informativo) (Figura 4C), e ter os estudantes como público-alvo (42\%) (Figura 4E), a maioria não utiliza a estrutura da Universidade em suas atividades (Figura 4D). $\mathrm{O}$ ambiente escolar tem papel importante para o aluno quanto à significação de diversas questões. É consenso que a Microbiologia continua sendo pouco explorada tanto no ensino fundamental quanto no ensino médio, sendo abordada nas escolas de maneira estritamente teórica (Palheta \& Sampaio, 2016). O ensino de Microbiologia contribui para que os alunos conheçam os microrganismos e sua influência sobre os seres e o meio ambiente, auxiliando no desenvolvimento de um senso crítico por parte dos estudantes. A Universidade costuma apresentar laboratórios mais bem estruturados do que a maioria das escolas, podendo oportunizar dinâmicas de ensino-aprendizagem diferenciadas. Mesmo sem recorrer à estrutura da Universidade, as práticas educativas foram tema da maior parte dos estudos (Figura 3). As atividades práticas são essenciais à compreensão, interpretação e assimilação dos conteúdos, permitindo que o aluno desenvolva a capacidade de observar, interpretar e inferir, formular hipóteses, fazer predições e julgamentos críticos a partir da análise de dados. Também despertam o interesse pela descoberta, da qual o aluno se torna agente, motivando-o a exercer a habilidade de explicar fenômenos com base em suas próprias experiências (Barbosa \& Barbosa, 2010).

A educação infantil necessita de maior atenção das ações de extensão universitária, visto que apenas dois dos 109 estudos analisados (Tabela S1) contemplaram esta etapa do ensino (Sousa et al., 2015; Strohschoen et al., 2016). Essa fase é marcada pela aguçada curiosidade e pelo início da transformação dos valores familiares, para a construção dos próprios valores. A escola passa a assumir papel destacado devido à sua função social e por sua potencialidade para o desenvolvimento de um trabalho sistematizado e contínuo (Secretaria de Educação Fundamental, 1997). A UNESCO adverte que a educação científica desde a idade precoce é de importância fundamental para o desenvolvimento humano, para criar a capacidade crítica, e para que tenhamos cidadãos informados e ativos perante a sociedade (Carvalho, 2005).

Predominam as ações de extensão baseadas na observação e na descrição (68\%) (Figura 4G). O diálogo, por exemplo, foi ferramenta utilizada por Da Silva e colaboradores (2011), que realizaram rodas de conversas sobre IST em escolas, e Oliveira e colaboradores (2014), que disseminaram informações sobre boas práticas para manicures. Ressalta-se, por outro lado, que a adoção de métodos sistematizados, bem como a realização de análises estatísticas, poderia elevar a qualidade das publicações que conectam a Microbiologia à Extensão Universitária.

A maior parte das ações de extensão com foco em Microbiologia oferece um retorno informativo às comunidades que atende em suas pesquisas (Figura $4 \mathrm{H}$ ). $\mathrm{O}$ conhecimento gerado por diversas esferas da sociedade, de maneira formal ou não, é fundamental para o desenvolvimento humano. A crescente inserção da ciência na sociedade incentiva a sua aceitação, uma vez que este contato propicia conhecer melhor o que se faz na ciência e o que dela resulta. Assim, torna-se crucial o modo pelo qual a atividade científica é transmitida, bem como as diferentes fontes de informação científica a que se tem acesso (Orlandini et al., 2015). A extensão universitária tem um papel importante na divulgação científica, na propagação de conhecimentos sobre Microbiologia e em sua contextualização social.

O presente estudo revelou diferentes aspectos das ações de extensão universitária centradas em temas da Microbiologia. Neste contexto, a comunidade externa à Universidade tem sido principalmente beneficiada nos 
campos da educação e da saúde, focando-se especialmente em doenças causadas por vírus. Estas ações caracterizam-se principalmente pelo teor observacional e descritivo, atentando-se para a necessidade da inserção de métodos que aumentem o rigor científico destes estudos, tais como a realização de entrevistas e questionários. Além disso, ressalta-se ainda a importância da Universidade pública para a continuidade destas ações, justificando-se os investimentos neste setor pelo efetivo retorno informativo e assistencial à coletividade (dentro ou fora do meio universitário). Acredita-se que estes dados podem ser utilizados como base para o aperfeiçoamento de futuras ações de extensão em Microbiologia.

\section{Considerações Finais}

Considerando-se a potencialidade da Extensão Universitária, em sua articulação com a pesquisa e o ensino, acredita-se que este levantamento bibliográfico contribua para que as ações voltadas à Microbiologia cresçam, se fortaleçam e aumentem ainda mais a sua significância social no cenário brasileiro.

\section{Contribuição de cada autor}

A pesquisadora B.R.L. concebeu, planejou, analisou e interpretou os dados, enquanto P.V. coordenou e orientou as ações referentes a este estudo.

\section{Referências}

Anthony, S. J., Epstein, J. H., Murray, K. A., Navarrete-Macias, I., Zambrana-Torrelio, C. M., Solovyov, A., \& Hosseini, P. (2013). A strategy to estimate unknown viral diversity in mammals. MBio, 4(5), e00598-13.

Barbosa, F. H., \& Barbosa, L. P. J. L. (2010). Alternativas metodológicas em microbiologia: Viabilizando atividades práticas. Revista de Biologia e Ciências da Terra, 10(2): 134-143.

Secretaria de Educação Fundamental. Brasil. (1997). Parâmetros Curriculares Nacionais: Primeiro e segundo ciclos: Meio ambiente, saúde. Brasília: MEC/SEF.

Buss, P. M. (2003). Uma introdução ao conceito de promoção da saúde. In D. Czeresnia, \& C. M. de Freitas (Eds.), Promoção da saúde: Conceitos, reflexões, tendências. (pp. 15-38). Rio de Janeiro: Editora FIOCRUZ.

Candeias, N. M. F. (1997). Conceitos de educação e de promoção em saúde: Mudanças individuais e mudanças organizacionais. Revista de Saúde Pública, 31, 209-213.

Carvalho, A. M. P. (2005). Introduzindo os alunos no universo das ciências. In J. Werthein, \& C. Da Cunha (Eds.), Educação científica e desenvolvimento: O que pensam os cientistas. (pp. 61-67). Brasília: UNESCO, Instituto Sangari.

Chauí. M. (2001). Escritos sobre a universidade. São Paulo: UNESP.

Da Fonseca, A. G., Bessa, A. B., \& De Souza Brito, T. N. (2011). Ações educativas para técnicos e auxiliares de laboratório de análises clínicas. Revista Extensão \& Sociedade, 5(3).

Da Silva, F. S., Batista Filho, R. C., \& De Sá Júnior, L. A. (2011). Oficinas de saúde preventiva nas escolas abertas de Natal/RN. Revista Extensão \& Sociedade, 5(3).

Da Silva, L. M., Da Rosa, M. C., Andersen, L. D. U., \& Vieira-Da-Motta, O. (2017). Vulnerabilidade sanitária e resistência microbiana: Sua implicação na qualidade de vida dos assentados rurais no Norte e Noroeste Fluminense. Revista de Extensão da UENF, 3(1), 79-92.

De Carvalho, I. T. (2010). Microbiologia Básica. Recife: EDUFRPE. Recuperado de http://pronatec.ifpr.edu.br/wpcontent/uploads/2013/06/Microbiologia_Basica.pdf 
De Magalhães Bastos, J. R., Camargo, L. M. A., De Castro, R. F. M., \& Da Silva, R. H. A. (2010). Projeto de melhoria de saúde bucal realizado em região amazônica (Monte Negro, RO). Revista de Cultura e Extensão USP, 3, 43-48.

Dos Santos, D. C. M., Da Silva, A. P. L., Da Costa, I. A. S., \& De Andrade Souza, G. P. V. (2017). Interação universidadeescola: Uso de jogos didáticos para conhecer e prevenir o Aedes aegypti. Revista Extensão \& Sociedade, 8(1), 57-68.

Fagundes, J. (1986). Universidade e compromisso social: Extensão, limites e perspectivas. Campinas: UNICAMP.

Farias, A. L. N., Schirmer De Mattos, J. L., \& Tiago, P. V. Diálogo entre os saberes popular e acadêmico no processo de transição agroecológica no Assentamento Chico Mendes III-PE. Extramuros - Revista de Extensão da UNIVASF, 5(2), 6479.

Ferreira, M. D. C. (2016). A formação emancipada e a Extensão Universitária: Uma experiência na orientação em saúde e prevenção de parasitoses. (Tese de Doutorado). Universidade Federal do Estado do Rio de Janeiro, Rio de Janeiro.

Fonseca, S. A., \& Lorenzo, H. C. de. (2004). Breve perfil das atividades de extensão nas unidades da UNESP, campus de Araraquara: Um enfoque na transferência de tecnologia e conhecimento. Revista Ciência em Extensão, 1(2), $112-119$.

Granato, T. M., Berriel, O. P., \& Intorne, A. C. (2015). Compostagem na Escola: Um estudo de caso na área rural do município de São Fidélis/RJ. Revista de Extensão da UENF, 2(1), 139-152.

Junior, P. P., Da Silva, M. D. C. S., Melo, R. D. C. C., De Lima, L. A., Araújo, K. S., De Oliveira Prado, I. G., \& Martin, J. G. P. A. (2018). Microbiologia no contexto da Agroecologia. Revista Brasileira de Extensão Universitária, 9(2), 75-82.

Korb, A., Teixeira, D. C., \& Mendonça Rodrigues, R. (2011). Os conhecimentos em biologia na educação em saúde. Revista de Biologia e Ciências da Terra, 11(1), 108-115.

Mesquita, M. O., Jantzen, M. M., Schons, M. Da S., \& Trevilaton, G. (2013). Atuação discente em ações de educação em saúde ambiental e vigilância sanitária em comunidade urbana reassentada. Revista da Extensão, 6, 59-64.

Moresco, T., Castro, T., Santos, E., \& Barbosa, N. B. (2015). Higiene pessoal: Contextualizando o ensino de microbiologia por meio da experimentação. In Anais do Salão Internacional de Ensino, Pesquisa e Extensão, 2015, Uruguaiana, 7. Uruguaiana: UNIPAMPA.

Oda, W. Y. (2012). A docência universitária em biologia e suas relações com a realidade das metrópoles amazônicas. (Tese de Doutorado). Universidade Federal de Santa Catarina, Florianópolis.

Oliveira, F. M., Dos Santos Alves, A., Santos, L. A., Santana, T. L. S., \& Silva, G. M. (2014). Projeto de Extensão "Faça as mãos, faça os pés: embeleze com segurança”. Revista Brasileira de Extensão Universitária, 5(2), 61-68.

Oliveira, F. M., Santana, T. L. S., Dos Santos, D. C. D. J., \& De Farias, L. H. S. (2016). Sistematização de práticas educativas relacionadas à higienização das mãos e microbiota para o ensino médio regular no contexto da extensão universitária. Interfaces-Revista de Extensão da UFMG, 4(1), 226-245.

Organização Mundial das Nações Unidas. ONU. (2015). Transformando Nosso Mundo: a Agenda 2030 para o Desenvolvimento Sustentável. Recuperado de https://nacoesunidas.org/pos2015/agenda2030

Orlandini, L. C., Casella, T., Batista, M. N., Machado, R. R. G., Akinaga, M. M., Chierotti M. C. M., De Moraes E. M., Scarpassa J. A., \& Rahal, P. (2015). Articulação ensino-pesquisa-extensão em Microbiologia: Difusão e popularização. In Congresso de extensão universitária da UNESP. (pp. 1-6). [ S. I.]: Universidade Estadual Paulista (UNESP).

Palheta, R. A., \& Sampaio, A. P. L. (2016). Atividades práticas sobre microrganismos no aprendizado do ensino médio. Revista Igapó - Revista de Educação Ciência e Tecnologia do IFAM, 10(1), 72-87.

Programa das Nações Unidas para o Desenvolvimento. PNUD. (2014). Atlas do Desenvolvimento Humano nas Regiões Metropolitanas Brasileiras. [S. I.]: PNUD. Recuperado de http://atlasbrasil.org.br/2013/pt/download/

Prado, I. C., Rodrigues, T. G., \& Khouri, S. (2004). Metodologia do ensino de microbiologia para ensino Fundamental e Médio. In Anais do Encontro Latino Americano de Iniciação Científica, 7, e Encontro Latino Americano de Pós-Graduação, 4. (pp. p.127-129). [S. I.]: Universidade do Vale do Paraíba. 
Rede Nacional de Extensão. RENEX. (2017). Lista de revistas acadêmicas em extensão no Brasil. Santo André: FORPROEX. Recuperado de http://www.ufmg.br/proex/renex/index.php/revistas

Sachet, E. U., \& De Arruda, M. P. (2018). Mãos limpinhas: Educação ambiental das famílias de catadores de resíduos sólidos urbanos. Revista de Extensão, 1(3), 32-42.

Sousa, R. A., Vilarinho N. M., Santiago, M. R., Lemos, J. F., Dos Santos, C. B., Medeiros, F. D. S., Júnior, S. C. D. S., \& D os Santos, K. R. (2015). Utilização de peças teatrais como auxílio na prevenção de parasitoses. Revista Ciência em Extensão, 11(1), 139-147.

Strohschoen, A. A. G., Spezia, A. P., Zuffo, S., Salvatori, R. U., \& Marchi, M. I. (2016). Atividades experimentais na educação infantil e ensinos fundamental e médio: Uma viagem ao mundo invisível. CCNExt-Revista de Extensão, 3(1), 643-648.

Teixeira, A. P. A., \& De Araújo, A. B. (2018). Ciência com micróbios: Uma proposta de mediação entre a universidade e a escola. Práticas em Gestão Pública Universitária, 2(2), 48-58.

Teixeira, P., \& Valle, S. (2010). Biossegurança: Uma abordagem multidisciplinar. Rio de Janeiro: SciELO -Editora FIOCRUZ.

Vieira, A. C. P. E., \& Oliveira, S. S. (2011). Educação ambiental e saúde pública: Uma análise crítica da literatura. Ambiente \& Educação, 16(1), 37-44.

Como citar este artigo:

Leite, B. R, \& Valente, P. A microbiologia e a extensão universitária. Revista Brasileira de Extensão Universitária, 11(1), 61-71. https://periodicos.uffs.edu.br/index.php/RBEU/article/ view/11123/pdf 